\title{
El desarrollo digital de los hogares e individuos. Una tipología de regiones europeas $^{1}$
}

\author{
R. González-Relaño (a), A. L. Lucendo-Monedero (b), F. Ruiz-Rodríguez (c) \\ (a) Universidad de Sevilla. rgrelano@us.es \\ (b) Universidad de Sevilla. angeluis@us.es \\ (c) Universidad de Sevilla. fruiz@us.es
}

\begin{abstract}
Resumen
El objetivo de este trabajo es caracterizar y clasificar las regiones europeas (NUTS2) en función de los niveles de desarrollo digital en los hogares e individuos. El desarrollo digital se mide a partir del acceso y uso que los hogares e individuos hacen de las Tecnologías de la Información y la Comunicación (TICs). La metodología se desarrolla en dos fases: 1) análisis factorial para identificar las variables clave que definen el desarrollo digital de hogares e individuos, a partir de los datos suministrados por Eurostat; y 2) partiendo del resultado anterior, análisis clúster para identificar y definir una tipología de regiones europeas. Se obtienen dos factores: "Disponibilidad de banda ancha y uso diario de comercio, banca y administración electrónica" y "Uso de redes sociales por banda ancha", que permiten definir siete tipos de regiones europeas con diferente grado de desarrollo digital.
\end{abstract}

Palabras claves: Acceso y uso de las TICs; hogares e individuos; regiones europeas; análisis multivariante; banda ancha y uso diario de Internet; redes sociales.

\section{INTRODUCCIÓN}

El crecimiento económico de las regiones y países se ve afectado directamente por el desarrollo, implantación y acceso a las Tecnologías de la Información y Comunicación (TIC). Se entiende por TICs el conjunto de herramientas, habitualmente de naturaleza electrónica, utilizadas para la recogida, almacenamiento, tratamiento, difusión y transmisión de la información. Esto agrupa tanto a los dispositivos físicos (equipos informáticos, redes de telecomunicación, terminales, etc.) como el software o aplicaciones informáticas que funcionan sobre estos equipos (INE, 2017). El proceso de desarrollo y difusión de estas tecnologías mejora la eficiencia y productividad de países y regiones incrementando no sólo las posibilidades de crecimiento económico sino también del bienestar social, ya que pueden mejorar el acceso a los servicios básicos, facilitar la conectividad y crear nuevas oportunidades. En definitiva contribuyen a mejorar la forma en que los individuos se comunican, interactúan y se relacionan entre sí y con sus gobiernos (Baller, S.et al, 2016). Pero a su vez, la dotación de infraestructura y la disponibilidad y uso de las TIC (Barzilai-Nahon, K. 2006; Van Dijk, J.A. 2006, Vu, K.M. 2011) refuerzan las desigualdades existentes (Helsper, E.J. 2012; Witte J.C. y Mannon, S.E. 2010) en la sociedad global de la información. Por ello surge la necesidad de medir y comprender el grado de desarrollo digital que surgen a partir de las diferencias entre tener accesibilidad a la red y usar ordenadores e internet (Yu, L. 2006; Sáinz Peña, R., 2017).

El estudio del desarrollo digital y las diferencias entre los distintos territorios comienza a abordarse por primera vez en la década de 1980, en relación con las carencias de infraestructuras de telecomunicaciones en los países en vías de desarrollo. Es en 1984 cuando se presenta el informe de la Unión Tecnológica Internacional (organismo adscrito a la ONU) que señala el importante desequilibrio tecnológico y de desarrollo digital existente entre los países avanzados y los países en desarrollo (ITU, 2003). Posteriormente, será en la década los noventa del siglo XX cuando los investigadores pretendan explicar la diferencia entre tener o no tener, usar o no usar ordenadores e internet por parte de los ciudadanos (Yu, L. 2006; Contreras-Cabrera, G. 2012). A finales del siglo

\footnotetext{
${ }^{1}$ Este trabajo forma parte de los resultados del Proyecto: "La Brecha Digital en la Unión Europea. Modelos Explicativos, Predictivos y Espaciales" (CSO2015-67662-P), financiado por el Ministerio de Economía y Competitividad (2016-18).
} 
pasado empiezan a desarrollarse investigaciones sobre la dotación de infraestructura, la disponibilidad y la asequibilidad de los ordenadores y el uso de Internet (Barzilai-Nahon, K. 2006; Van Dijk, J. A 2006). Sin embargo, el desarrollo digital se entendía inicialmente de forma binaria (Cruz-Jesus F. et al, 2012) y se limitaba a distinguir entre tener y no tener acceso a las TIC. Esta concepción era útil para describir las desigualdades sociales y tecnológicas, pero era una clasificación binaria muy reductiva, imprecisa e inexacta.

Actualmente, el estudio del desarrollo digital se aborda desde diferentes niveles de análisis: los individuoshogares (individual), las empresas (organizacional) y el estado (global). El nivel individual distingue entre las personas que están dispuestas a integrar las TIC en su vida cotidiana y las que están a la zaga en el acceso y uso de nuevas tecnologías. Por otro lado, el desarrollo digital también se ha abordado desde la perspectiva espacial a diferentes escalas geográficas (Pick J.B. y Nishida, T., 2015): a escala mundial (Yates D.J. et al, 2011; Pick, J.B. y Azari, R. 2011), macrorregional (Kiiski, S. y Pohjola, M. 2002; Lengsfeld, J.H. 2001; Igari, N., 2014), y estatal y/o zonas económicas dentro de las naciones (Vicente, R.M. y López, A.J. 2011; Nishida T. et al, 2014), y en menor medida, estudios a escala regional (NUTS2 ${ }^{2}$ ) para el conjunto de Europa (Vicente, R.M. y Lopez, A.J., 2011).

Por otro lado, el desarrollo digital ha sido estudiado más en términos de acceso (infraestructuras) a las TIC y que en relación al uso (habilidades) de Internet. Sin embargo, la importancia del acceso a internet comenzó a cuestionarse cuando la conectividad a la red mediante banda ancha y los dispositivos digitales se hicieron más frecuentes (Scheerder, A. et al 2017). Esto puso de manifiesto que el desarrollo digital de los hogares e individuos pasó a estar condicionado por el uso de internet que realizan las personas (Brandtzaeg, P.B. et al, 2017, Hargittai, E. 2010) y no por nivel de accesibilidad a internet que es prácticamente generalizado, sobre todo en los países desarrollados (Sáinz Peña, R., 2017). En relación al uso de internet, los estudios generalmente se han centrado en los términos de frecuencia y el tipo de actividades (Scheeder, A. et al, 2017). Estas investigaciones han revelado que, aunque la búsqueda de información general en internet ha sido uno de los usos más comunes (Purcell, K. 2011), el aumento de la popularidad de las redes sociales ha cambiado los tipos de uso hacia aquellos que buscan una mayor interacción social (Van Deursen, A. J. et al, 2015).

En base a estos precedentes y ante la ausencia de estudios geográficos y regionales, este trabajo pretende identificar qué variables de acceso y uso de las TICs definen actualmente el desarrollo digital en hogares e individuos de las regiones europeas para establecer una tipología de las mismas que muestre el grado de desarrollo digital a escala regional.

\section{METODOLOGÍA}

\subsection{Fuentes, variables utilizadas y unidad de análisis}

Los datos que se han empleado en este trabajo proceden de la encuesta "Uso de las TIC en los hogares y por individuos" (isoc_i) de Eurostat para el año 2017. Dicha encuesta proporciona datos sobre el acceso y uso de las tecnologías de la información y la comunicación para dos unidades estadísticas: los hogares (todos los hogares privados con al menos un miembro en el grupo de edad de 16 a 74 años); y los individuos (personas de entre 16 y 74 años). La encuesta proporciona datos de los estados miembros de la UE, países candidatos, e Islandia y Noruega. Además, Eurostat proporciona estadísticas desagregadas por regiones de residencia (NUTS, apartado "isoc_reg"). Las 16 variables utilizadas se detallan en el Cuadro 1 agrupadas por áreas y unidad de medida (porcentajes de hogares y/o de personas).

El área de referencia escogida para el análisis regional del desarrollo digital de Europa en hogares e individuos es el nivel NUTS2 de las regiones de los 28 países del UE más República de Macedonia, Noruega, Islandia, Suiza y Turquía. Sin embargo, las variables de la encuesta "Uso de las TIC en los hogares y por individuos" de Eurostat enumeradas en el Cuadro 1 están desglosadas a nivel de NUTS2 para todos los países indicados anteriormente excepto para Alemania, Grecia, Polonia, Reino Unido y Turquía. Para solventar la falta de datos a escala regional de estos países y, por tanto, evitar realizar un análisis regional incompleto del desarrollo digital en hogares e individuos, se incluye en el estudio las regiones NUTS1 de estos países para los cuales Eurostat sí ofrece datos de las variables seleccionadas en el Cuadro 1. En consecuencia, para llevar a cabo esta investigación se ela-

\footnotetext{
${ }^{2}$ La clasificación NUTS (Nomenclature of territorial units for statistics) es el sistema jerárquico de división de la UE Fuente: Eurostat (2017c) <http://ec.europa.eu/eurostat/documents/345175/7451602/NUTS-map-ES.pdf>.
} 
boró una base de datos con 16 variables de uso de TICs en los hogares e individuos (Cuadro 1) para 242 regiones europeas (190 NUTS2 y 52 NUTS1 ${ }^{3}$ ).

Cuadro 1 - Variables empleadas en el análisis.

\begin{tabular}{|c|c|}
\hline VARIABLE Y UNIDAD DE MEDIDA & ABREVIATURA \\
\hline \% Hogares que tienen conexión a internet & H_ACCESO_Casa \\
\hline \% Hogares que tienen conexión a internet por banda ancha & H_ACCESO_Banda ancha \\
\hline \% Hogares que con acceso a internet & H_ACCESO_Intenet \\
\hline$\%$ Individuos que usan internet diariamente & IND_USO_Diario \\
\hline $\begin{array}{l}\text { \% Individuos que participan en redes sociales (creando perfiles de usurario, } \\
\text { enviando mensajes u otras participaciones en Facebook, Twitter, etc.) }\end{array}$ & IND_USO_Redes sociales \\
\hline \% Individuos que usan internet para banca electrónica & IND_USO_Banco \\
\hline \% Individuos que usan internet para vender bienes o servicios & IND_USO_Vender \\
\hline \% Individuos que usan internet siempre & IND_USO_Siempre \\
\hline \% Individuos que acceden a internet fuera de casa o en el trabajo & IND_ACCESO_Fuera \\
\hline $\begin{array}{l}\text { \% Individuos que interactuaron con las autoridades públicas (en los últimos } \\
\qquad 12 \text { meses) }\end{array}$ & IND_INTER_Autoridades \\
\hline $\begin{array}{l}\text { \% Individuos que interactuaron con las autoridades públicas para enviar } \\
\text { formularios cumplimentados (en los últimos } 12 \text { meses) }\end{array}$ & IND_INTER_Enviar formularios 12 meses \\
\hline $\begin{array}{l}\text { \% Individuos que compraron algún producto o servicio a través de Internet } \\
\text { en los últimos } 3 \text { meses }\end{array}$ & IND_COMPRA_Online_3 meses \\
\hline $\begin{array}{l}\text { \% Individuos que compraron algún producto o servicio a través de Internet } \\
\text { en el último año }\end{array}$ & IND_COMPRA_Online último año \\
\hline $\begin{array}{l}\text { \% Individuos que solicitaron bienes o servicios, a través de Internet, para uso } \\
\text { privado, hace más de un año o nunca }\end{array}$ & IND_COMPRA_Online nunca o hace más de 1 año \\
\hline $\begin{array}{l}\text { \% Individuos que reservaron a través de internet alojamiento de vacaciones } \\
\text { (en los últimos } 12 \text { meses) }\end{array}$ & IND_COMPRA_Viajes \\
\hline \% Individuos que compraron a través de internet a otros países de la EU & IND_COMPRA_A otros paises UE \\
\hline
\end{tabular}

Fuente: Elaboración propia.

\subsection{Análisis factorial}

El estudio conjunto de todos los aspectos que contempla el concepto de desarrollo digital en los hogares e individuos de las regiones europeas demanda el empleo de técnicas estadísticas multivariantes. Este tipo de métodos estadísticos permiten analizar las interdependencias entre el acceso a las TICs, el uso diario de internet en diversos dispositivos en el hogar y fuera de él para el comercio y otras actividades personales. Autores como Cruz -Jesus, F. et al. (2012, 2016) consideran que el análisis factorial (FA) es una técnica estadística especialmente adecuada para el análisis del desarrollo digital, dada la c

omplejidad del estudio de las TIC en la sociedad y la economía.

Para conocer cómo se interrelacionan las variables de uso de las TIC en los hogares y en individuos, se ha llevado a cabo un análisis factorial de tipo exploratorio y por el método de componentes principales mediante el paquete informático estadístico IMB SPSS v23. Mediante el análisis exploratorio se pretende obtener un número óptimo de factores y la estructura factorial que defina el desarrollo digital en hogares e individuos de las regiones europeas. Por su parte, el método de componentes principales es el más adecuado cuando se pretende establecer alguna teoría o modelo de manera inicial ya que busca encontrar el menor número de factores que expliquen la mayor cantidad de varianza o información posible (Frías-Navarro, D. y Pascual Soler, M, 2012). Con este fin se optó por obtener una matriz factorial rotada por el método Varimax.

\subsection{Análisis Clúster}

La identificación de tipologías de regiones europeas implica agruparlas en conjuntos definidos por la homogeneidad de características comunes de acceso y uso cotidiano de las TIC en hogares e individuos. La tipología de las regiones europeas se ha obtenido mediante el análisis clúster a partir de los resultados obtenidos en el AF. EI

\footnotetext{
${ }^{3}$ Por falta de datos se omitieron en el análisis final 7 NUTS2 y 2 NUTS1.
} 
empleo de métodos de análisis multivariantes de conglomerados es de gran utilidad en los casos que se busca resolver problemas de identificación y clasificación en unidades homogéneas a partir de varias variables. Autores como Lebart, L. et al. (1995) y de Pardo, C.E. y Del Campo, P.C. (2007) aconsejan la combinación del AF y del análisis clúster en dos etapas: primero un análisis de conglomerados jerárquicos $\mathrm{y}$, posteriormente, un análisis de $\mathrm{K}$ medias. Este procedimiento soluciona diversos inconvenientes de las técnicas de clustering como pueden ser la clasificación a partir de pocas variables significativas o no correlacionadas (basadas en los factores del AF); o el problema derivado de las diferencias de escala de las variables utilizadas (que se evitaría al utilizar como variables de agrupamiento las puntuaciones $z$ de los factores normalizados).

Mediante el análisis de k-medias se obtuvieron los valores medios de cada grupo (centros de los conglomerados finales) a partir de los cuales se calculan los porcentajes de hogares e individuos que usan TICs en cada región para los dos factores. Cada clúster agrupa regiones que presentan porcentajes similares de desarrollo digital en hogares e individuos en los 2 factores, estableciendo una tipología de desarrollo digital de las regiones europeas. Así, los clústeres que tienen mayor porcentaje de hogares/individuos en los 2 factores tienen un mayor desarrollo digital (y, al contrario).

\section{RESULTADOS}

\subsection{Factores que determinan el desarrollo digital en los hogares y por individuos de las regiones europeas}

El análisis factorial exploratorio se realizó con las 16 variables de uso de las TIC en los hogares y por individuos de las regiones europeas. El AF válido incluye 14 variables y las medidas de adecuación de las variables introducidas (determinante, test de KMO y test de esfericidad de Bartlett, Cuadro 2) muestran que las variables están altamente correlacionadas entre sí y, por tanto, el resultado del AF es correcto. En efecto, el determinante de la matriz de correlaciones es muy bajo (1,89E-012); el test de Bartlett permite contrastar la hipótesis de nulidad de ausencia de correlación entre las variables; y el valor del índice adecuación muestral KMO es igual o superior a 0,80 (en concreto, 0,847) tal y cómo recomienda Kaiser (1970).

La elección del número de factores finales se hizo en función de la Varianza total explicada y el método Kaiser como puede observarse en el Cuadro 3. Se obtuvieron 2 factores porque éstos suman mayor cantidad de información posible (la varianza total explicada acumulada entre los 2 factores es de casi el $80 \%$, cumpliendo el criterio de Pearson) y además son los 2 componentes con un autovalor propio superior a 1 (de acuerdo con el método de Kaiser).

Cuadro 2 - KMO y prueba de Bartlett.

\begin{tabular}{|c|c|c|}
\hline \multicolumn{2}{|c|}{$\begin{array}{c}\text { Medida de adecuación muestral de } \\
\text { Kaiser-Meyer-Olkin. }\end{array}$} & 0,870 \\
\hline & Chi-cuadrado aproximado & 6105,11 \\
\cline { 2 - 3 } $\begin{array}{c}\text { Prueba de esfericidad de } \\
\text { Bartlett }\end{array}$ & gl & 105 \\
\cline { 2 - 3 } & Sig. & 0,000 \\
\hline
\end{tabular}

Determinante de la matriz de correlaciones $=1,89 \mathrm{E}-012$.
Cuadro 3 - Varianza total explicada.

\begin{tabular}{|c|c|c|c|}
\hline \multirow{2}{*}{ Componente } & \multicolumn{3}{|c|}{ Autovalores iniciales } \\
\cline { 2 - 4 } & Total & \% del varianza & $\%$ acumulado \\
\hline 1 & 9,678 & 69,130 & 69,130 \\
\hline 2 & 1,438 & 10,274 & 79,404 \\
\hline 3 & 0,764 & 5,454 & 84,858 \\
\hline 4 & 0,515 & 3,682 & 88,540 \\
\hline 5 & 0,374 & 2,673 & 91,213 \\
\hline 6 & 0,360 & 2,572 & 93,785 \\
\hline 7 & 0,294 & 2,099 & 95,883 \\
\hline 8 & 0,213 & 1,524 & 97,407 \\
\hline 9 & 0,129 & 0,924 & 98,330 \\
\hline 10 & 0,078 & 0,556 & 98,886 \\
\hline 11 & 0,064 & 0,459 & 99,345 \\
\hline 12 & 0,044 & 0,312 & 99,657 \\
\hline 13 & 0,036 & 0,261 & 99,918 \\
\hline 14 & 0,011 & 0,082 & 100,000 \\
\hline
\end{tabular}

Método de extracción: Análisis de componentes principales. 
En el Cuadro 4 muestra la matriz de componentes rotados por el método de Varimax en la que podemos observar las correlaciones entra las variables y los 2 factores obtenidos. La interpretación de los mismos es la siguiente:

El factor 1 aporta el 69,13\% de la varianza. Está formado por 12 variables relacionadas positivamente con:

i) La disponibilidad de Internet y la capacidad de acceso por banda ancha en los hogares (H_ACCESO_Casa y H_ACCESO_Banda ancha).

ii) El uso diario de Internet (IND_USO_Diario y IND_USO_Siempre).

iii) Las actividades realizadas por motivos particulares como: banca electrónica (IND_USO_Banco), venta de bienes o servicios (venta directa, mediante subastas, eBay, etc., IND_USO_Vender); compra a través de Internet en los últimos meses, en concreto algún producto o servicio (IND_COMPRA_Online_3 meses y IND_COMPRA_Online último año), de servicios de alojamiento de vacaciones (hotel, apartamento, etc., IND_COMPRA_Viajes) o a vendedores de otros países del Unión Europea (IND_COMPRA_A otros paises UE); el contacto o interacción con las administraciones o servicios públicos por Internet por motivos particulares (IND_INTER_Autoridades) y/o para enviar formularios cumplimentados (como, p. ej., presentar la declaración del renta u otros impuestos (IND_INTER_Enviar formularios 12 meses), y

iv) Las personas que accedieron a Internet fuera del hogar o del trabajo (IND_ACCESO_Fuera).

Esto indica que, en las regiones europeas, la disponibilidad de acceso a internet por banda ancha en los hogares está asociada al uso diario de internet por motivos particulares de los individuos para el comercio electrónico, banca electrónica y para relacionarse con los entes públicos a través de administración electrónica ya sea en los propios hogares o fuera de éste. Este factor se puede denominar "Disponibilidad de banda ancha y uso diario de comercio, banca y administración electrónica".

El factor 2 representa solo el 10,27\% de la varianza, y establece una relación entre dos variables: utilización de conexión a internet por banda ancha en los hogares (H_ACCESO_Banda ancha) con el uso de redes sociales (IND_USO_Redes sociales). Se denominaría "Uso de redes sociales por banda ancha".

\subsection{Tipología de regiones europeas según desarrollo digital.}

La clasificación de las regiones europeas con el análisis clúster, a partir de los resultados obtenidos en el AF, por el procedimiento del vecino más lejano y el método de Ward con la distancia euclídea al cuadrado permite apreciar a 7 grupos diferenciados de regiones europeas a partir del dendograma obtenido. Por su parte, mediante el análisis de k-medias se obtuvieron los valores medios de cada grupo (centros de los conglomerados finales, Cuadro 5); y se calculó la media del porcentaje de hogares e individuos de los dos factores para el conjunto de regiones europeas de cada clúster, lo que permite determinar el grado de desarrollo digital de las mismas. Como se avanzaba en metodología, cada clúster agrupa regiones que presentan porcentajes similares de desarrollo digital en hogares e individuos en los 2 factores. Si se calcula la media del valor de los porcentajes de hogares/ individuos de los 2 factores para cada grupo (Cuadro 5) se puede establecer una tipología jerarquizada de desarrollo digital de las regiones europeas (Mapa 1). En consecuencia, las regiones europeas (NUTS2) se ordenan de mayor a menor nivel de desarrollo digital de la siguiente manera:

Clúster 1: Regiones con máximo nivel de desarrollo digital. Es el grupo de regiones que presentan mayores 
porcentajes de desarrollo digital de hogares/individuos con una media del 73,7 \%. Lo componen 48 regiones: la mayor parte de las NUTS2 de Noruega, Suecia, Finlandia, Reino Unido, regiones de Alemania occidental, Países Bajos e Islandia. En consecuencia, estas regiones presentan los valores más altos de hogares/individuos tanto en el factor 1 "Disponibilidad de banda ancha y uso diario de comercio, banca y administración electrónica" (63,3 \%); como en el factor 2 "Redes sociales por banda ancha" (84,3\%).

Clúster 2: Regiones con alto nivel de desarrollo digital (70,3\% de media de hogares/individuos). Está formado por 20 regiones: la británica de Northen Ireland, la noruega de Hedmark og Oppland, la sueca de Sydsvrige, la finlandesa Etelä-Suomi, alemana de Hamburg y Berlín, las regiones belgas de Anterwepen, Wlaams-Brabant y Brabant Vallon, la francesa de lle de France y las regiones de Suiza (excepto una), Dinamarca y Estonia. Estas regiones se caracterizan por tener porcentajes más altos de hogares/individuos que usan TICs en los dos factores por detrás de las regiones del Clúster 1: un 61,9\% de hogares/individuos de media para el factor 1 ("Disponibilidad de banda ancha y uso diario de comercio, banca y administración electrónica); y un 78,7\% para el factor 2 "Redes Sociales por banda ancha".

Clúster 3. Regiones con nivel alto-medio de desarrollo digital $(64,6 \%)$ ligeramente por encima de la media europea (62,8\% de media). Es el grupo más numeroso de NUT2s (60) que comprende a las regiones de Irlanda, España (excepto Galicia), Bélgica (excepto las mencionadas en el cluster 2), gran parte de Alemania, sobre todo las regiones orientales, regiones Austriacas, extendiéndose por las regiones del sur de la Republica Checa, Eslovenia, norte de Hungría y Eslovenia, y Letonia. El clúster se sitúa en el tercer nivel de desarrollo digital con casi un 50\% de hogares/ individuos con "Disponibilidad de banda ancha y uso diario de comercio, banca y Administración electrónica"; y porcentajes altos de hogares/individuos que hacen "Uso de redes sociales por banda ancha" (77,9\%).

Clúster 4: Regiones con nivel medio-bajo de desarrollo digital con déficit en el factor 2 ("Uso de redes sociales por banda ancha"). Está compuesto por 21 regiones, todas las francesas menos Isle de France. Estas regiones presentan un nivel en torno a la media europea de desarrollo digital (59,9\% de media de hogares/individuos) que se basa fundamentalmente en un porcentaje alto-medio $(53,6 \%)$ de hogares/individuos en el factor 1 ("Disponibilidad de Acceso a banda a ancha y uso diario de comercio, banca y administración electrónica". Por el contario, los porcentajes de hogares/individuos en el "Uso de redes sociales por banda ancha" (factor 2) se alejan 10 puntos de la media europea (sólo un 66,2\%).

Clúster 5: Regiones con medio-bajo nivel de desarrollo digital con déficit en el factor 1 ("Disponibilidad de banda ancha y uso diario de comercio, banca y administración electrónica"), con valores en ambos factores por debajo de la media europea (un 57,9\% de hogares/individuos). Está formado por 31 regiones: Galicia (España), Región Centro, Algarve e islas de Portugal, regiones del norte y centro de Italia, resto de regiones checas, húngaras y eslovenas no incluidas en el cluster 3, las regiones occidentales rumanas (Nord-Vest, Vest) y la región capital de Bucaresti-fov, la región búlgara de Yugozapaden y las turcas de Ankara y Konya. Estas regiones ocupan la quinta posición en desarrollo digital sustentándose en valores altos del factor 2 ya que el 76,4 \% de los hogares/ individuos usan las redes sociales por banda ancha. Por el contrario, sólo el 39,3\% de ellos en estas regiones tienen "Disponibilidad de banda ancha y uso diario de comercio, banca y administración electrónica" (factor1).

Clúster 6: Regiones con nivel bajo de desarrollo digital (por debajo de la media europea, con el 53,2\% de hogares/individuos) con déficit del factor 2. Este último grupo está formado por 16 regiones de la Europa del Este (la región alemana de Bradenburg, el resto de regiones polacas no incluidas en el grupo 6, Stredné Slovesko en Eslovaquia, Sud-Vest Ottania en Rumanía y las regiones turcas del Noreste, Centro-Este y Sudeste de Anatolia y del Este del Mar Negro). Las regiones de este grupo tienen porcentajes de los hogares/individuos por debajo de la media fundamentalmente en el factor "Uso de redes sociales por banda ancha" (es el grupo de regiones que más se aleja de toda Europa de la media en 7 puntos porcentuales). Igualmente, los porcentajes de hogares/individuos son bajos en el factor 1 "Disponibilidad y acceso a banda ancha para el uso diario de comercio, banca y administración electrónica" (36,9\%).

Clúster 7. Regiones de nivel bajo de desarrollo digital de hogares/individuos con un mayor déficit en el factor 1 ("Disponibilidad de acceso a internet y uso diario de comercio, banca y administración electrónica"). Este conglomerado lo forman 37 regiones: regiones portuguesas e italianas no incluidas en el cluster 4, regiones grie- 
gas, de Macedonía, 3 regiones polacas del sur y este del país (Wschodni y Makroregion Pólnocno-Zachodni), regiones bosnias, búlgaras (excepto Yugozapaden) y las regiones turcas occidentales y centrales (Aegean, Marmara, del Mar Negro, Central Anatolia, etc.). Al igual que las regiones del grupo anterior, tienen valores alejados de la media en los dos factores, pero en este clúster el mayor déficit está en el factor 1 (casi 9 puntos porcentuales por debajo de la media con un 32,9\% de los hogares/individuos); mientras que el factor 2 ("Uso de redes sociales por banda ancha) tiene valores relativamente altos (72,5\% de hogares/individuos).

Cuadro 5 - Tipología de Desarrollo Digital de las Regiones

\begin{tabular}{|c|c|c|c|c|c|c|c|}
\hline Jerarquía & $\begin{array}{l}\text { NIVEL DE DESARROLLO } \\
\text { DIGITAL }\end{array}$ & $\begin{array}{l}\text { Centro de conglo- } \\
\text { merados finales } \\
\text { Factor1 }\end{array}$ & $\begin{array}{l}\text { Centro de conglo- } \\
\text { merados finales } \\
\text { Factor2 }\end{array}$ & $\begin{array}{c}\text { Nivel medio } \\
\text { de desarrollo } \\
\text { digital }\end{array}$ & $\begin{array}{l}\text { Nivel medio } \\
\text { de Factor1 }\end{array}$ & $\begin{array}{l}\text { Nivel medio } \\
\text { de Factor2 }\end{array}$ & $\mathrm{N}^{\circ}$ regiones \\
\hline 1 & MÁXIMO & 1,05 & 0,83 & 73,7 & 63,2 & 84,3 & 48 \\
\hline 2 & ALTO & 1,16 & $-0,19$ & 70,3 & 61,9 & 78,7 & 20 \\
\hline \multirow[t]{2}{*}{3} & ALTO-MEDIO & $-0,06$ & 0,49 & 64,6 & 50,0 & 77,9 & 60 \\
\hline & Media & & & 62,8 & 49,0 & 76,6 & 233 \\
\hline 4 & MEDIO-BAJO F2* & 0,96 & $-2,21$ & 59,9 & 53,6 & 66,2 & 21 \\
\hline 5 & MEDIO-BAJO F1* & $-1,00$ & 0,45 & 57,9 & 39,3 & 76,4 & 31 \\
\hline 6 & BAJO F2* & $-0,61$ & $-1,49$ & 53,2 & 36,9 & 69,6 & 16 \\
\hline \multirow[t]{2}{*}{7} & BAJO F1* & $-1,34$ & $-0,25$ & 52,7 & 32,9 & 72,5 & 37 \\
\hline & & \multicolumn{2}{|c|}{ Puntaciones factoriales } & \multicolumn{3}{|c|}{ \% de hogares e individuos } & \\
\hline
\end{tabular}

Fuente: Elaboración propia.

${ }^{*} \mathrm{~F} 1$ y $\mathrm{F} 2$ : factor 1 y factor 2 , respectivamente.

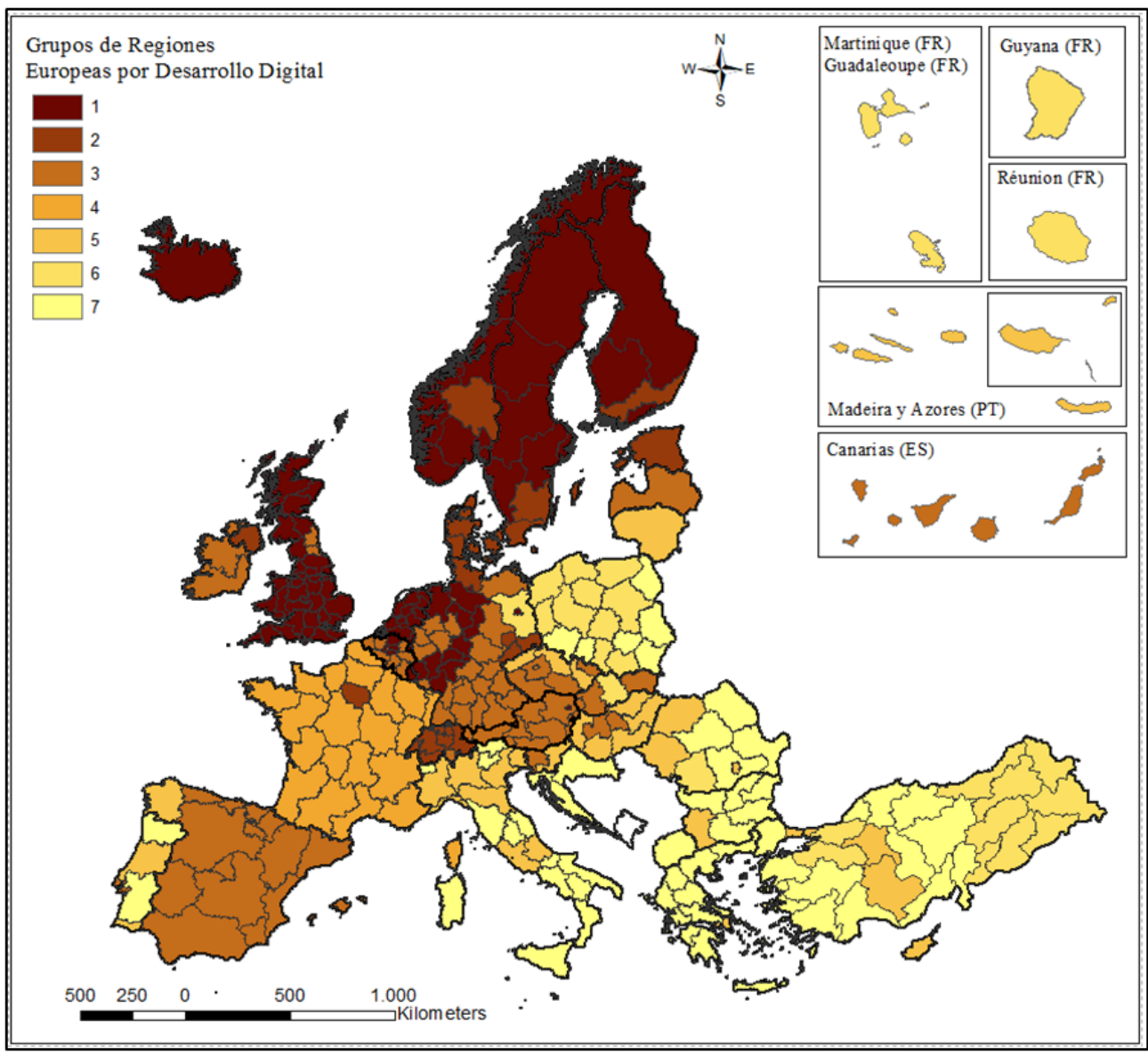

Mapa 1 - Grupos de regiones europeas según el grado de desarrollo digital. Fuente: Elaboración propia. 


\section{CONCLUSIONES}

El uso que los hogares/individuos de las regiones europeas hacen de las TICs viene determinado por la disponibilidad y el acceso a la banda ancha como muestran los resultados del AF. La conexión por banda ancha es el componente más importante y común en el comportamiento respecto al uso de las TICs de los hogares e individuos. Sin embargo, aunque las infraestructuras de banda ancha están extendidas en toda Europa (el $83 \%$ de media de hogares e individuos en las regiones de estudio), las diferencias en los niveles de desarrollo digital entre regiones europeas depende el porcentaje de hogares/individuos que usan a diario las TICs para el comercio, banca y administración electrónica (factor 1); mientras que el uso de internet para participar en las redes sociales (factor 2) al estar más generalizado, no incide en los niveles de desarrollo digital de las regiones europeas.

Igualmente, los resultados de este estudio muestran que más de la mitad de las regiones europeas han alcanzado un nivel medio y alto de desarrollo digital (el 55\% de las mismas). No obstante, en su conjunto, las regiones presentan un comportamiento heterogéneo y jerarquizado en el nivel de desarrollo digital, lo que pone de manifiesto la existencia de desequilibrios espaciales en el nivel de uso y acceso a las TICs en los hogares/ individuos, y, por lo tanto, la existencia de brecha digital entre regiones. Así, por un lado, el mayor acceso y uso de las TICs avanzadas se realiza en las regiones del Norte y Centro de Europa. Mientras las regiones del sur y este de Europa registran menores porcentajes de hogares/individuos que utilizan las TICs respecto a las regiones del norte del continente. Y, por otro, las diferencias en el desarrollo digital en Europa son menores en relación al uso de redes sociales que en el uso diario de comercio, banca y administración electrónica. Desde la perspectiva de la Geografía Económica, las diferencias de desarrollo digital entre regiones europeas que se han identificado en este estudio siguen el comportamiento espacial del modelo de desarrollo centro-periferia (Ruiz-Rodríguez, F. et al, 2018, Billon, M. et al, 2016) imperante en innovación (Jordá-Borrell, R., et al, 2015)

No obstante, esta investigación presenta una serie de limitaciones. En primer lugar, el número de variables incluidas en el análisis (14) es limitado debido a la escasa disponibilidad de datos a escala regional NUTS2 para Europa. Por otra, si bien el uso del análisis clúster permite identificar y comparar a nivel agregado el nivel de desarrollo digital entre los 7 grupos de regiones europeas (clústers), sería conveniente analizar de forma más detallada las diferencias de comportamiento entre las regiones europeas, lo que podría realizarse mediante la elaboración de un indicador complejo que permita medir y comparar el desarrollo digital de las regiones europeas.

Las conclusiones de este trabajo sugieren profundizar en el estudio del desarrollo digital de las regiones europeas a nivel individuos/hogares. Así, sería de interés conocer las causas de las diferencias regionales de desarrollo digital. Ello conllevaría medir la incidencia de diversas variables sobre el desarrollo digital, tanto de tipo socioeconómico como geográficas (estructura empresarial regional, actividades de I+D e innovación, capacidades TICs, sensibilización digital, nivel educativo, renta, políticas digitales y otros aspectos endógenos del territorio, etc.). Ello puede abordarse mediante la aplicación de técnicas de regresión que permite conocer cuáles son los factores que explican las diferencias regionales del uso de las TICs en los hogares/individuos.

\section{BIBLIOGRAFIA}

BALLER, S., DUTTA, S., \& LANVIN, B. (2016). Global Information Technology Report 2016. Ouranos.

BARZILAI-NAHON, K. (2006). Gaps and bits: Conceptualizing measurements for digital divide/s. The information society, 22(5), 269-278.

BILLON, M., LERA-LOPEZ, F., \& MARCO, R. (2016). ICT use by households and firms in the EU: links and determinants from a multivariate perspective .Review of World Economics,152(4), 629-654.

BRANDTZAEG, P. B., HEIM, J., \& KARAHASANOVIĆ, A. (2011). Understanding the new digital divide-A typology of Internet users in Europe. International journal of human-computer studies, 69(3), 123-138.

CONTRERAS-CABRERA, G. (2012). Asociacionismo en red para la integración sociodigital en Andalucía. Revista de Estudios Andaluces, (29), 105-126.

CRUZ-JESUS, F., OLIVEIRA, T., \& BACAO, F. (2012). Digital divide across the European Union. Information \& Management, 49 (6), 278-291.

CRUZ-JESUS, F., VICENTE, M. R., BACAO, F., \& OLIVEIRA, T. (2016). The education-related digital divide: An analysis for the EU-28. Computers in Human Behavior, 56, 72-82.

EUROSTAT (2018): http://ec.europa.eu/eurostat/web/digital-economy-and-society/data/database. Acceso el 10 enero 2018.

FRÍAS-NAVARRO, D., \& PASCUAL SOLER, M. (2012). Prácticas del análisis factorial exploratorio (AFE) en la investigación sobre conducta del consumidor y marketing. Suma Psicológica, 19(1).

HARGITTAI, E. (2010). Digital na ( $t$ ) ives? Variation in internet skills and uses among members of the "net generation". Sociological inquiry, 80(1), 92-113. 
HELSPER, E. J. (2012). A corresponding fields model for the links between social and digital exclusion. Communication theory, 22 (4), 403-426.

IGARI, N. (2014). How to successfully promote TIC usage: A comparative analysis of Denmark and Japan. Telematics and Informatics, 31(1), 115-125.

INE (2017): Encuesta sobre el uso de TIC y comercio electrónico en las empresas Metodología, Spain, Madrid (2017). http:// www.ine.es/daco/daco42/comele/metocor.pdf,Acceso 25 marzo 2018

INTERNATIONAL TELECOMMUNICATION UNION -ITU- (2003): International Telecommunication Union -ITUWorld telecommunication development report 2003: Access indicators for the information society. International Telecommunications Union,Geneva, Switzerland (2003). http://www.itu.int/net/wsis/tunis/newsroom/stats/WorldTelecomDevelopmentReport -2003_E.pdf, Acceso 10 enero 2018.

JORDÁ BORRELL, R., RUIZ RODRÍGUEZ, F., \& GONZÁLEZ RELAÑO, R. (2015). Factors and taxonomy of technology purchase (TP) by internationalized innovative companies in peripheral European regions. Papers in Regional Science, 94(S1).

KIISKI, S., \& POHJOLA, M. (2002). Cross-country diffusion of the Internet. Information Economics and Policy, 14(2), 297-310.

LEBART, L., MORINEAU, A. \& PIRON, M. (1995), Statisitique exploratoire multidimensionnelle, Dunod, Paris.

LENGSFELD, J. H. (2011). An econometric analysis of the sociodemographic topology of the digital divide in Europe. The information society, 27(3), 141-157.

NISHIDA, T., PICK, J. B., \& SARKAR, A. (2014). Japan's prefectural digital divide: A multivariate and spatial analysis. Telecommunications policy, 38(11), 992-1010.

PARDO, C. E., \& DEL CAMPO, P. C. (2007). Combinación de métodos factoriales y de análisis de conglomerados en R: el paquete FactoClass. Revista colombiana de estadística, 30(2).

PICK, J. B., \& AZARI, R. (2011). A global model of technological utilization based on governmental, business-investment, social, and economic factors. Journal of Management Information Systems, 28(1), 49-84.

PICK, J. B., \& NISHIDA, T. (2015). Digital divides in the world and its regions: A spatial and multivariate analysis of technological utilization. Technological Forecasting and Social Change, 91,1-17.)

PURCELL, K. (2011). Search and email still top the list of most popular online activities. Pew Internet \& American Life Project, 9, 1 $-15$.

RUIZ-RODRÍGUEZ, F., LUCENDO-MONEDERO, A. L., \& GONZÁLEZ-RELAÑO, R. (2018). Measurement and characterisation of the Digital Divide of Spanish regions at enterprise level. A comparative analysis with the European context. Telecommunications Policy, Vol. 42, Issue 3, April 2018, Pages 187-211.

SÁINZ PEÑA, R. (2017): Sociedad digital en España, 2017. Fundación Telefónica y Editorial Ariel.

SCHEERDER, A., VAN DEURSEN, A., \& VAN DIJK, J. (2017). Determinants of Internet skills, uses and outcomes. A systematic review of the second-and third-level digital divide. Telematics and informatics.

VAN DEURSEN, A. J., \& HELSPER, E. J. (2015). A nuanced understanding of Internet use and non-use among the elderly. European journal of communication, 30(2), 171-187.

VAN DIJK, J. A. (2006). Digital divide research, achievements and shortcomings. Poetics, 34(4-5), 221-235.

VICENTE, M. R., \& LÓPEZ, A. J. (2011). Assessing the regional digital divide across the European Union-27. Telecommunications Policy, 35(3), 220-237.

VU, K. M. (2011). TIC as a source of economic growth in the information age: Empirical evidence from the 1996-2005 period. Telecommunications Policy, 35(4), 357-372.

WITTE, J. C., \& MANNON, S. E. (2010). The internet and social inequalitites. Routledge.

YATES, D. J., GULATI, G. J., \& WEISS, J. W. (2011, January). Different paths to broadband access: The impact of governance and policy on broadband diffusion in the developed and developing worlds. In System Sciences (HICSS), 2011 44th Hawaii International Conference on (pp. 1-10). IEEE.

YU, L. (2006). Understanding information inequality: Making sense of the literature of the information and digital divides. Journal of Librarianship and Information Science, 38(4), 229-252. 